\title{
La necesidad de especialización en Fisioterapia en España
}

\author{
The need for specialization in Physiotherapy in Spain
}

La visión de la ER.WCPT sobre educación en Fisioterapia en el año 2020 obedece a las necesidades de los profesionales y la sociedad, y a la responsabilidad de los organismos europeos para dar respuesta a las mismas.

En los últimos 20 años, la ER-WCPT ha desarrollado un proceso de especialización para los fisioterapeutas. El concepto de especialización es consecuencia de la cooperación entre las organizaciones miembros en el seno de la ER-WCPT, y resultado del trabajo de una serie de reuniones y conferencias celebradas sobre la educación post básica y la especialización. El concepto también se basa en las relaciones entre la especialización y el empleo, teniendo en cuenta la migración libre en Europa para los fisioterapeutas.

El proceso de especialización fue iniciado hace años por los colectivos de fisioterapeutas de muchos países europeos, como Austria, Dinamarca, Finlandia, Alemania, Italia, Holanda, etc. En los documentos Interim Repart an Recomendations of Specialisation for Physiotherapits within the European Region of the $W_{C P T^{1}}$ y Recomendatian for a Proposed Model for the Development of Specialisation within Physiotherapy in the European Region of the $W C P T^{2}$, aprobados en reunión general de la ER-WCPT, se recogen múltiples datos y recomendaciones sobre el establecimiento de modelos de especialización en Fisioterapia. En concreto, se hace referencia a que la especialización puede ser un proceso tanto informal como formal, y se expone la necesidad de diferenciar entre un experto clínico en un tema específico (Specific Clinical Expert), elevada realización académica (High Academic Achievement) y especialización en Fisioterapia (Specialization in Physiotherapy) ${ }^{2}$.

Según los resultados obtenidos mediante encuestas, la mayoría de los países miembros proponen alcanzar la formación especializada empleando el nivel de master ${ }^{1}$. No obstante, la ER-WCPT establece que se debe garantizar que las vías de especialización incluyan el desarrollo clínico y académico y reconoce que los programas de máster con diferentes contenidos clínicos especializados dan nuevas oportunidades para ampliar conocimiento y las posibilidades de desarrollo de la carrera profesional para los especialistas en Fisioterapia ${ }^{3}$.

La visión de la ER-WCPT es la excelencia en el nivel educativo de entrada y posterior cualificación en toda Europa, que sea internacionalmente reconocido, y basado en el consenso de educadores, estudiantes, empresarios, opinión pública, otros profesionales de la salud y partes interesadas.

La educación debe satisfacer las necesidades de Fisioterapia en la sociedad del futuro con el fin de mejorar la salud, la capacidad funcional y el bienestar de la población. Los programas educativos de alta calidad conducen a una mayor empleabilidad de los fisioterapeutas europeos; esto a su vez mejorará la libre circulación y el derecho de establecimiento de los fisioterapeutas de toda Europa ${ }^{4}$.

Para la ER-WCPT, el desarrollo profesional continuo se centra en la especialización, y los documentos elaborados al respecto proporcionan información a los fisioterapeutas, educadores, autoridades y organizaciones competentes de la Unión Europea, además de la pauta de la WCPT para el desarrollo profesional continuo de calidad para los fisioterapeutas ${ }^{4}$.

En España, la necesidad de especialización en Fisioterapia es notoria desde diferentes ámbitos. Por una parte, el número de profesionales fisioterapeutas en España se ha incrementado de forma muy rápida en los últimos años, pasando de 26.343 registros en 2006 a 42.490 en 2014, con una tasa de variación anual de crecimiento aproximada- mente del $6,5 \%$.

Así mismo, existen en España 55 centros universitarios que imparten el título de grado de Fisioterapia (30 en universidades públicas y 25 en privadas) que se encuentran representadas por la Conferencia Nacional de Decanos de Facultades de Fisioterapia (CNDFF), y el ámbito profesional está organizado en 17 colegios, uno por cada comunidad autónoma, integrados en el Consejo General de Colegios de Fisioterapeutas de España. Además, la Asociación Española de Fisioterapeutas (AEF) como estructura de 
representación científica y desarrollo profesional es la institución que representa a los fisioterapeutas españoles en el seno de la WCPT.

La realidad de la Fisioterapia es la consecuencia de la profunda madurez y amplitud alcanzada por el cuerpo de conocimientos de la disciplina y desarrollo de competencias profesionales en el campo asistencial. Por todo ello, se ha promovido en el seno del colectivo el necesario debate sobre el establecimiento de especialidades en Fisioterapia para aumentar la calidad asistencial y aumentar la investigación en el campo asistencial.

Respecto a las posibles vías de formación de fisioterapeutas especialistas, caber señalar que, a diferencia de lo que ocurre en otras profesiones sanitarias como la medicina y la enfermería, que tienen una presencia cuantitativa elevada y transversal en los hospitales y centros de salud del Sistema Nacional de Salud (SNS), la Fisioterapia queda limitada a desarrollar sus funciones desde los servicios centralizados de rehabilitación, lo que limita su contacto con el resto de servicios del hospital y dificulta el desarrollo y la implementación de una prestación asistencial especializada. Además, el número de fisioterapeutas pertenecientes a las plantillas de los centros sanitarios del SNS es cuantitativamente bajo. Esta realidad, condiciona que no pueda desarrollarse un adecuado sistema de encuadramiento y tutorización destinado a la formación especializada por el modelo exclusivo de residencia. Por otro lado, varias de las posibles especialidades de alto interés para la Fisioterapia, como el deporte o el envejecimiento, no se ofertan por el SNS.

En España, el 89,6\% de los fisioterapeutas tienen menos de 45 años, y la mayor parte de la asistencia en Fisioterapia se oferta en el ámbito liberal del ejercicio profesional. Estos profesionales deberían disponer de un sistema de formación especializada que se adapte a sus necesidades, lo que significa que puedan compatibilizar su puesto de trabajo con el programa de formación. La formación por el sistema característico de residencia impide esta posibilidad al exigir exclusividad en la contratación del residente. Por lo tanto, se plantea una residencia parcial (prácticas clínicas) en el ámbito público (SNS, centros sociosanitarios, etc.) y privado.

Los fisioterapeutas españoles adquieren sus competencias orientadas a la especialización en un marco de desarrollo profesional continuo, mediante la formación de posgrado en métodos específicos o másteres universitarios, con el fin de ofrecer a sus pacientes o usuarios servicios basados en la evidencia y de alta calidad.

El desarrollo profesional de los especialistas precisa de un reconocimiento de la especialidad por parte de las instituciones sanitarias en los diferentes países y en la Región Europea. El reconocimiento por parte de las instituciones sanitarias de los diferentes países de determinadas especialidades de Fisioterapia llevará aparejado que los fisioterapeutas especialistas trabajen principalmente en esas áreas específicas de la práctica clínica o la enseñanza, y participen en la investigación y la evaluación y el desarrollo de los servicios relevantes para la práctica clínica.

Además, la creación de un mayor número de puestos de fisioterapeutas especialistas proporcionará a la profesión los líderes clínicos que faciliten el desarrollo de la especialidad y aborden temas clave para mantener y mejorar la salud de los ciudadanos (como, por ejemplo, la eficacia clínica y la práctica basada en la evidencia).

En España la tasa actual de fisioterapeutas por cada 1.000 habitantes es de 9,1, pero se desconoce si, por especialidades, la tasa es proporcionada para dar respuesta a los problemas de salud de la población en esos ámbitos específicos.

Teniendo en cuenta esta realidad y para responder a estas necesidades, la AEF ha elaborado un documento con una propuesta de especialización ${ }^{5}$. Se trata de modelo de especialización vía máster oficial, en consonancia con los países de la ER-WCPT, que evita las dificultades del modelo de residencia exclusiva y puede garantizar el cumplimiento de los máximos criterios de calidad.

Los másteres oficiales se presentan como una posibilidad real de especialización profesional alternativa al modelo exclusivo de residencia. Pero para su correcto funcionamiento debe garantizarse que los elementos nucleares del proyecto docente estén adecuadamente identificados y atendidos, destacando los que han de permitir la necesaria y correcta articulación entre los centros docentes responsables de los programas y prácticas clínicas.

El Real Decreto 1393/2007 ${ }^{5}$, de ordenación de las enseñanzas universitarias oficiales, en su artículo 10, relativo a las enseñanzas de máster, recoge: «Las enseñanzas de máster tienen como finalidad la adquisición por el estudiante de una formación avanzada, de carácter especializado o multidisciplinar, orientada a la especialización académica o profesional, o bien a promover la iniciación en tareas investigadoras». Por otro lado, en el punto 3 se refiere a la denominación de los títulos de máster para garantizar que identifique con precisión la actividad formativa y «no conduzca a error sobre su nivel o efectos académicos ni a confusión sobre su contenido y, en su caso, efectos profesionales» .

Así mismo, el artículo 15.4 abre la posibilidad de organizar másteres oficiales de orientación profesional al establecer que «cuando se trate de títulos que habiliten para el ejercicio de actividades 
profesionales reguladas en España, el Gobierno establecerá las condiciones a las que deberán adecuarse los correspondientes planes de estudios, que además deberán ajustarse, en su caso, a la normativa europea aplicable. Estos planes de estudios deberán, en todo caso, diseñarse de forma que permitan obtener las competencias necesarias para ejercer esa profesión. A tales efectos la Universidad justificará la adecuación del plan de estudios a dichas condiciones» ${ }^{5}$.

En consonancia con lo expuesto, el modelo de especialización profesional vía másteres oficiales desarrollado por la $\mathrm{AEF}^{6}$, que ha sido entregado en el Ministerio de Sanidad Español, contempla una formación que combina la docencia universitaria con la realización de un ejercicio clínico sistematizado y supervisado en unidades clínico docentes acreditadas, que permitan al especialista en formación adquirir las competencias profesionales propias de la especialidad. Este modelo se presenta como una posibilidad real alternativa al modelo de residencia que existe en la actualidad en España en el SNS para otros profesionales sanitarios, como médicos o enfermeras.

El Proyecto sobre las especialidades en Fisioterapia ${ }^{6}$ cuenta por parte de la CNDFF, y profesional con su aceptación por parte de diferentes Colegios Profesionales de Fisioterapeutas de España, establece el marco conceptual, ideológico y argumental en el que debería encuadrarse el proceso de regulación de las especialidades en Fisioterapia en nuestro país, con el propósito de agilizar un proceso coherente de su reconocimiento por parte de la Administración.

El documento de las especialidades de la $\mathrm{AEF}^{6}$ establece que para conseguir un modelo de formación especializada oficial y con reconocimiento legal y profesional resulta necesario que este se desarrolle respetando algunas características, como las siguientes:

- Que posea una denominación igual en todo el territorio nacional.

- Que aborde un cuerpo de conocimientos específico y con unos contenidos iguales en todos los programas que se oferten.

- Que garantice una formación clínica con la misma programación formativa y carga horaria en todos los programas que se oferten.

- Que los sistemas de evaluación de los especialistas en formación sean homogéneos en todos los programas que se oferten.

- Que los centros clínicos en los que se imparta la práctica profesional estén acreditados según los mismos criterios para todos los programas que se oferten.

- Que las competencias profesionales adquiridas estén oficialmente reconocidas y reservadas en todo el territorio nacional.

- Que el sistema de acreditación de los programas se realice a través de la ANECA (u otra agencia de calidad universitaria).

Por ello, la formación de máster deberá garantizar las cualificaciones que tienen como finalidad la adquisición por el estudiante de una formación avanzada, de carácter especializado, orientada a la especialización profesional, que corresponde con en el nivel de máster (nivel 3 del Marco Español de Cualificación de la Educación Superior (MECES) ${ }^{7}$, incluyendo conocimientos profesionales avanzados y altamente especializados, junto al progreso de la autonomía clínica correspondiente al desarrollo profesional y de su especialización. Así, los modelos formativos que se diseñen deberán incluir todos los recursos necesarios para garantizar las cualificaciones profesionales correspondientes a un determinado ámbito clínico especializado dentro de un programa de formación.

\section{Bibliografia}

1. Interim Report on Recomendation of Specialisation for Physiotherapits within the European Region of the WCPT. General Meeting 25-27 May 2006, Sibenik, Croatia.

2. Recomendation for a Proposed Model for the Development of Specialisation within Physiotherapy in the European Region of the WCPT. General Meeting of the European Region of the WCPT. 13-15 May 2004, Chipre.

3. Policy Statement on Physiotherapy Education of the ER-WCPT. General Meeting of the European Region of the WCPT. 8-10 May 2014, Copenhagen, Denmark 2014.

4. A European Vision of CPD Including Specialisation. General Meeting of the European Region of the WCPT. 810 May 2014, Copenhagen, Denmark

5. Real Decreto 1393/2007, de 29 de octubre, por el que se establece la Ordenación de las Enseñanzas Universitarias Oficiales (BOE de 30 de octubre de 2007).

6. Fernández R, Souto S, González L, Lista A, Gómez A, Asociación Española de Fisioterapeutas. Proyecto de Especialidades en Fisioterapia. Madrid: Asociación Española de Fisioterapeutas; 2015. 
7. Real Decreto 102712011, de 15 de julio, por el que se establece el Marco Español de Cualificaciones para la Educación Superior (BOE de 3 de agosto de 2011).

\author{
A. Gómez Conesa ${ }^{\mathrm{a}, \mathrm{b}}$, R. Fernández Cervantes $^{\mathrm{c}}$ y S. Souto Camba ${ }^{\mathrm{d}, \mathrm{e}}$ \\ ${ }^{a}$ Departamento de Fisioterapia, Universidad de Murcia, Murcia, España \\ ${ }^{\mathrm{b}}$ Asociación Española de Fisioterapeutas \\ ${ }^{c}$ Decanato Facultad de Fisioterapia, Universidad de A Coruña, La Coruña, España \\ ${ }^{\mathrm{d}}$ Departamento de Fisioterapia, Universidad de A Coruña, La Coruña, España \\ ${ }^{\mathrm{e}}$ Executive Committee ER-WCPT
}

